\title{
Article
}

\section{The reliability and validity of the Chinese version of the Metacognitions about Health Questionnaire in college students}

Dai, Lisha, Bailey, Robin and Deng, Yunlong

Available at http://clok.uclan.ac.uk/21345/

Dai, Lisha, Bailey, Robin and Deng, Yunlong (2018) The reliability and validity of the Chinese version of the Metacognitions about Health Questionnaire in college students. Quality of Life Research . ISSN 0962-9343

It is advisable to refer to the publisher's version if you intend to cite from the work. http://dx.doi.org/10.1007/s11136-017-1780-5

For more information about UCLan's research in this area go to http://www.uclan.ac.uk/researchgroups/ and search for <name of research Group>.

For information about Research generally at UCLan please go to http://www.uclan.ac.uk/research/

All outputs in CLoK are protected by Intellectual Property Rights law, including Copyright law. Copyright, IPR and Moral Rights for the works on this site are retained by the individual authors and/or other copyright owners. Terms and conditions for use of this material are defined in the policies page. 


\title{
The reliability and validity of the Chinese version of The
}

\section{Metacognitions about Health Questionnaire in college stu-}

\section{dents}

\author{
Lisha Dai $^{1}$, Robin Bailey ${ }^{2}$, Yunlong Deng ${ }^{1}$ \\ ${ }^{1}$ Department of Psychiatry, the Third Xiangya Hospital, Central South University, \\ No. 138 Tongzipo Road, Yuelu District, Changsha, Hunan 410013, China. \\ ${ }^{2}$ School of Health, BB235, University of Central Lancashire, Preston, Lanca- \\ shire PR1 2HE, UK. \\ Correspondence should be addressed to Yunlong Deng: deng0087@126.com
}

(1)

\section{Abstract}

Purpose In order to explain the potential mechanism that might motivate and maintain health anxiety (HA), researchers have developed several measures to assess the level of HA and to identify related cognitions and personality features. However, such instruments typically measure general metacognitions (e.g., the Metacognition Questionnaire-30, MCQ-30), thereby compromising the degree of sensitivity and specificity of measurement as applied to HA-related metacognitions. To address that issue, the Metacognitions about Health Questionnaire (MCQ-HA) was designed especially for measuring metacognitive beliefs specific to HA. Because a Chinese version of MCQ-HA may be helpful in improving our understanding of HA in a Chinese population, in the current study we sought to develop a Chinese version of the MCQ-HA (CMCQ-HA).

Methods We translated the MCQ-HA into Chinese with consideration of cultural diversity. For evaluation of its validity and stability, a sample of 1290 Chinese college students answered the CMCQ-HA, the Short Health Anxiety Inventory, the MCQ-30 and the Neuroticism scale of the Eysenck Personality Questionnaire. 292 students of them answered the CMCQ-HA twice.

Results Good internal consistency $(\alpha=0.81)$ and test-retest reliability (ICC $=0.70)$ of the CMCQ-HA was presented. Exploratory and confirmatory factor analyses indicated a three-factor structure: beliefs about biased thinking, beliefs that thoughts can cause illness, 
1 and beliefs that thoughts are uncontrollable. Convergent validity, divergent validity and in-

2 cremental validity all were acceptable. Measurement invariance across gender was estab-

3 lished.

4 Conclusions The CMCQ-HA shows promise for the measurement of specific HA-related 5 metacognitions in Chinese populations.

6 Keywords Health anxiety, Metacognition, Reliability and validity, Chinese population

\section{Introduction}

Health anxiety (HA) is a psychological condition that involves excessive worry about one's own health in the absence of relevant physical illness [1]. People with elevated levels of HA may experience excessive distress and functional impairment, and utilize medical services at a greater frequency [2]. Such experiences are not uncommon in either general population or clinical samples, and have attracted increasing attention in the health field [3]. Epidemiological studies indicate that the prevalence rate of HA is $3.4 \%$ in community samples and even higher in primary practice samples [4,5]. Furthermore, HA is usually comorbid with physical disease and other psychological disorders, and has been shown to complicate disease diagnosis and to elevate treatment difficulty [4]. Recently, there has been a lot of progress in the mechanism of HA. A meta-analysis supported a consistent link between the personality trait neuroticism and various clinical disorders [6], while other work found a strong correlation between neuroticism and severe HA [7]. Cognitive-behavioral theory is currently the most

21 influential model for conceptualizing and treating HA [8], and studies have identified cogni22 tive variables that play a role in the development and maintenance of HA, including dysfunctional illness beliefs, catastrophic misinterpretation, and somatosensory amplification [9-11].

24 A range of instruments were developed to estimate those variables, such as the Whiteley Index [12] and Health Anxiety Questionnaire [13] for level of HA, and the Health Cognitions

26 Questionnaire [14] and Somatosensory Amplification Scale [11] for HA-related cognitive variables.

28 There is also evidence suggesting that cognitive and personality variables may not fully ex- 
1 plain the development and persistence of HA [15]. Metacognitive Theory (MCT) [16, 17],

2 which is based on the Self-Regulatory Executive function (S-REF) model, argues that HA

3 usually results from extended and repetitive negative thinking about illness, and that such

4 negative thinking is the consequence of dysfunctional metacognitive beliefs [15]. The role of

5 metacognitive beliefs in HA has been studied in a range of studies, including HA as a

6 cross-sectional and longitudinal predictor of disorder [15-18]. In some of those studies the ge-

7 neric Metacognition Questionnaire-30 (MCQ-30), a shortened version of the Metacognition

8 questionnaire (MCQ), was used to measure metacognitive beliefs [19]. However, as the

9 MCQ-30 is designed for measuring general metacognition in psychological disorders, none of

10 the items are specifically HA-related. Another study used the Metacognitions about health

11 anxiety (MCHA) scale, which was revised based on the MCQ; however, this is an un-

12 published scale, and knowledge of its psychometric properties is very limited [20]. In order to

13 further explore the psychological mechanism of HA and promote more appropriate treatments

14 for it, an improved measure of HA-related metacognitive beliefs is essential. In 2015, Bailey

15 and Wells developed a 14-item questionnaire, the Metacognitions Questionnaire-Health Anxi-

16 ety (MCQ-HA) [20] to measure metacognitive beliefs specific to HA. The measure captures

17 three different HA-related metacognition constructs: beliefs about biased thinking, beliefs that

18 thoughts can cause illness and beliefs that thoughts are uncontrollable. The MCQ-HA has

19 been shown to have good reliability and validity, and a useful measurement of HA-related

20 metacognitive beliefs $[15,16]$.

21 In 2012, Rachman first proposed the concept of health anxiety disorders (HAD), and regarded

22 HAD as an anxiety disorder just like panic disorder, social phobia, general anxiety disorder

23 [21]. It's worth noting that no scale yet can measure both anxiety and health anxiety [22]. In

24 China, a plenty of measures for anxiety disorders were established but a few scales were de-

25 veloped to assess HA or hypochondriasis which is deemed as the extreme form of HA [23].

26 Most studies in this field focus on the cognitive characteristics and treatment of hypochondri-

27 asis. The Whiteley Index is the most widely used measurement in Chinese population, but the

28 full information about its psychometric properties in mainland China is not clear [24]. And a 
1 majority of HA-related measurements available in China each are concentrating on one cog-

2 nitive aspect of HA, such as the Anxiety Sensitivity Index, Health Anxiety Inventory and so

3 on [25]. The cognitive structure of HA or hypochondriasis is still under controversy [15], so

4 more measures to assess different aspects of HA are needed to developed for either research-

5 ers or clinic practice. To our best knowledge, there was no research exploring the role of met-

6 acognition in developing HA in Chinese population. It may be the case that there is no appro-

7 priate Chinese scale to measure it. Several researches suggest that cultural diversity has ex-

8 tensive influence on cognition, emotion and motivation [26]. Across different culture contexts,

9 people may hold particular culturally-related traditional views that influence their cognitions

10 regarding health. Accordingly, metacognitions may be culture-based and the development of a

11 measurement for HA-related metacognitions in China can be helpful in improving our under-

12 standing of metacognitions and HA. It is unknown whether the MCQ-HA which is a validated

13 scale for HA can be a good measurement of HA-related metacognitions in China. We aimed

14 to address this issue through the current research. The main purpose of our study was to

15 translate the MCQ-HA into Chinese and test the reliability and validity of CMCQ-HA in

16 measuring HA-related metacognitions in the context of Chinese culture.

\section{Methods}

\section{Participants}

19 Participants were recruited by convenience sampling from a university medical school in the 20 Hunan Province in China. Questionnaires containing information about the study were dis21 tributed during a class break. 1290 undergraduate students in 13 classes voluntarily agreed to 22 participate and gave informed consent. In the present study, we acquired 1191 fully completed 23 questionnaires; the effective return ratio is $92.3 \%$. Two weeks later, a random sample drawn 24 from three classes comprised of a total of 292 students were chosen for a second testing ses25 sion; of them, 268 completed the CMCQ-HA. That second sample was used for the test-retest 26 reliability analysis; effective return ratio was $91.8 \%$. Information about age and gender were obtained from all participants. There was no academic or other reward for their participation.

28 The study protocol received full approval from the local ethics committee. 
3 The original MCQ-HA consists of 14 items and has three subscales for assessing three types

4 of HA-related metacognitive beliefs: beliefs about biased thinking (5 items), beliefs that

5 thoughts can cause illness (5 items) and beliefs that thoughts are uncontrollable (4 items).

6 Items are rated on a 4-point Likert scale ranging from 1 (do not agree) to 4 (agree very much)

7 [20]. Subscale score for Beliefs about biased thinking and Beliefs that thoughts can cause ill-

8 ness ranges from 5 to 20 , subscale score for Beliefs that thoughts are uncontrollable ranges

9 from 4 to 16 . The total MCQ-HA score ranges from 14 to 56 with higher score indicating

10 higher levels of unhelpful HA-related metacognitive beliefs. Permission to use that measure

11 was obtained from Robin Bailey who was the original author of MCQ-HA. Taking reference

12 of guideline from Beaton et al, the Chinese version of MCQ-HA was created [27]. First, two

13 psychological researchers translated the MCQ-HA into Chinese. Then the translations were

14 synthesized into one. After that, two psychology professors examined the translation for sur-

15 face-level relevance to the construct of interest and each item's suitability for a Chinese popu-

16 lation. Next, the Chinese version of the items was back-translated by a professional bilingual translator who had not read the original MCQ-HA. That version was reviewed and modified

18 by the author of the MCQ-HA until it expressed exactly the same meanings as the original 19 measure. Because the resulting description of Item 3 "I will be punished for thinking I am in 20 good health ", was not a good fit for Chinese culture, and has a religious connotation, it was 21 modified as follows: "There will be something bad to happen for thinking I am in good 22 health". This modification was approved by Dr. Bailey. A pilot test was conducted with 25 23 participants. There were no reports of misunderstandings.

24 The Short form of Health Anxiety Inventory (SHAI)

25 The SHAI measures the severity of health anxiety and contains 18 items [13]. In current study, 26 it was employed for exploration of convergent validity, divergent validity and incremental 27 validity. The Chinese version of SHAI consists of two factors: illness likelihood (IL) with 14 
1 items, and negative consequences (NC) with 4 items. There are four statements ranging

2 from 0 (I do not) to 3 (I spend most of my time) in each item. The total SHAI score

3 ranges from 0 to 54; and higher scores indicating increased health anxiety. The SHAI

4 showed good psychometric propertied in Chinese population. The Cronbach's alpha

5 of its total and subscales were 0,742 (total), 0.743 (IL) and 0.788 (NC) [28]. In this

6 study, the Cronbach's alphas of SHAI total and its subscales were 0.805 (total),

$7 \quad 0.788(\mathrm{IL}), 0.664(\mathrm{NC})$.

8 The Metacognition Questionnaire-30 (MCQ-30)

9 The MCQ-30 is widely used to measure metacognitive beliefs [19]. In the current study, we 10 chose to use it for an incremental validity study. The 30 items is a refinement of the original 11 MCQ [29]. The Chinese version of MCQ-30 shown good psychometric properties and con12 sists of five specific subscales: positive beliefs about worry, negative beliefs about uncontrol13 lability of thoughts and danger, cognitive confidence, beliefs about the need to control 14 thoughts and cognitive self-consciousness [30]. The response options for of each item ranged 15 from 1 (do not agree) to 4 (agree very much). The MCQ-30 total was ranging from 30 to 120 16 and indicating different tendency in generic metacognitive beliefs. In this study, the 17 Cronbach's alphas for the MCQ-30 were 0.857 for total items, and ranged from 0.674 to 0.822 18 for subscale items.

19 Neuroticism scale of the Eysenck Personality Questionnaire-Revised: Short Form (EPQ-R-N)

20 EPQ-R is a well-known personality assessment instrument with 100 items and 4 subscales: 21 extraversion, neuroticism, psychoticism and lie; Eysenck et al developed a revised version 22 that consists of 48 items [31]. The Chinese version of the EPQ-R includes 48 items and 4 23 subscales as does the original version [32]. The response to each item is "yes" or "no" with 24 scored 1 or 0 . In order to better explain the results, the raw score of each subscale should be 25 converted to standard score. The neuroticism subscale assesses the feature of emotional re26 sponse. In the present study we only use the neuroticism subscale for evaluating divergent 27 validity; Cronbach's alpha for this subscale was 0.818 . 


\section{Data analysis}

\section{Item analysis and reliability}

3 A corrected item-total correlation were estimated for homogeneity and the recommended cri-

4 terion was above 0.3 [33]. Reliability of the CMCQ-HA was evaluated by investigating its

5 internal consistency and test-retest reliability. Internal consistencies of total score and three

6 subscales of the CMCQ-HA were assessed. For the test-retest reliability, the intraclass class

7 correlation coefficient (ICC) was considered to be more suitable compared to Pearson's cor-

8 relation coefficients [34]; accordingly, the ICC results are reported.

\section{Validity}

10 Exploratory factor analysis (EFA) and confirmatory factor analysis (CFA) was conducted to 11 explore the factor structure. To do those analyses we randomly divided the participants into two equal groups ( $\mathrm{n}=596$ and $\mathrm{n}=595$ ) by the SPSS algorithm. In Group 1, we conducted the EFA for the purpose of identifying the latent variables by SPSS 22.0. For factor extraction,

14 principal axis factoring (PAF) and promax rotation were used, while the results of parallel analysis, eigenvalues and scree plot were inspected. In the pattern matrix, the primary factor loading higher than 0.32 was acceptable [35]. The parallel analysis was conducted by SPSS syntax script from O'Connor [36]. In Group 2, we conducted the CFA with maximum likelihood estimation by Mplus 7.0. According to the criteria proposed by Hu and Bentler [37], four commonly used indices were chosen: comparative fit index (CFI), root mean squared error of approximation (RMSEA), standardized root mean square residual (SRMR) and the Tuck-

21 er-Lewis index (TLI). The convergent validity, divergent validity, and criterion-related validi22 ty of this scale were evaluated by Pearson's correlation coefficients for the CMCQ-HA score, the CSHAI score, and the EPQ-R score. A $P$-value less than 0.05 were considered as statisti-

24 cally significant. The incremental validity of CMCQ-HA beyond MCQ-30 for HA was examined with hierarchical multiple regression.

\section{Gender invariance}

27 Multigroup CFA was performed for the gender invariance of CMCQ-HA with Mplus 7.0. 
1 There were four models tested for measurement invariance across gender: configural invari-

2 ance, metric invariance, scalar invariance, and strict invariance [38].

\section{Results}

\section{Descriptive statistics of study subjects}

5 In the 1191 participants, no data was missing for all scales. All the items fell within the rec-

6 ommended range reflecting skew and kurtosis coefficients that should not be above 3 and 10 ,

7 respectively [39]. The distribution of each item was close to normality. As shown in Table 1, a

8 moderate floor effect was shown for the beliefs about biased thinking subscale (22.67\%) and

9 beliefs that thoughts are uncontrollable subscale (17.8\%) [40]. For the beliefs that thoughts

10 can cause illness subscale and total score, the floor effects were $5.46 \%$ and $2.35 \%$, respec-

11 tively. The observed ceiling effects were negligible. Participants were 726 women (61\%) and

12465 men (39\%). Age range 17-24, with 19.33 \pm 1.32 (mean \pm S.D.) years; no significant age

13 difference was observed between the two gender groups ( $t=0.555 ; 95 \%$ confidence interval

$14[\mathrm{CI}]:-0.112-0.200 ; p=0.579)$. Mean and standard deviation for age and each questionnaire are

15 presented in Table 1.

16 Table 1 Descriptive statistics for age and measurements

\begin{tabular}{lccccccc}
\hline & $\mathrm{N}$ & $\operatorname{Mean}(\mathrm{SD})$ & $\mathrm{Min}$ & $\mathrm{Max}$ & $95 \% \mathrm{CI}$ & \%Min & $\% \mathrm{Max}$ \\
\hline Age & 1191 & $19.33(1.32)$ & 17 & 24 & & & \\
CMCQHAT & 1191 & $24.40(6.17)$ & 14 & 55 & {$[24.05-24.75]$} & 2.35 & 0.08 \\
CMCQHAT-R & 286 & $22.45(4.51)$ & 14 & 43 & {$[21.90-22.99]$} & 1.12 & 0.37 \\
BI & 1191 & $10.24(3.25)$ & 5 & 20 & {$[10.06-10.43]$} & 5.46 & 0.67 \\
BT & 1191 & $7.29(2.35)$ & 5 & 20 & {$[7.15-7.42]$} & 22.67 & 0.34 \\
BU & 1191 & $6.87(2.47)$ & 4 & 16 & {$[6.73-7.01]$} & 17.80 & 0.34 \\
SHAI & 1191 & $11.73(5.32)$ & 0 & 35 & {$[11.43-12.04]$} & & \\
MCQ30 & 1191 & $69.50(11.73)$ & 31 & 116 & {$[68.83-70.17]$} & & \\
EPQ & 1191 & $5.62(3.37)$ & 0 & 12 & {$[5.43-5.82]$} & & \\
\hline
\end{tabular}

17 Notes: CMCQHAT = Metacognitions about Health total; CMCQHAT-R = Metacognitions 
1 about Health total at second time; BI = Beliefs that Thoughts can Cause Illness; BT $=$ Beliefs

2 about biased Thinking; BU = Beliefs that Thoughts are Uncontrollable; SHAI $=$ Short Health

3 Anxiety Inventory; MCQ-30 = Metacognition questionnaire-30; EPQ = Neuroticism subscale

4 of the Eysenck Personality Questionnaire-Revised: Short Form.

\section{$5 \quad$ Item analysis and reliability}

6 To test the homogeneity of the scale, corrected item-total correlations ranging from $0.35-0.54$

7 were examined and all above the recommended cut off 0.3 [33]. As shown in Table 2, correla-

8 tions between each of the CMCQ-HA subscales and the total score ranged from $0.73-0.80$

9 ( $p<0.01)$. The inter-correlations between subscales ranged from 0.35-0.43 $(p<0.01)$, suggest-

10 ing that these subscales are related to each other but assess independent aspects of HA-related

11 metacognitions. Internal consistency was evaluated with Cronbach's alpha. A Cronbach's al-

12 pha of lower than 0.6 or 0.50 was seen as unacceptable for total score or subscale scores, re-

13 spectively [41]. This index of CMCQ-HA total score was 0.81 . The Cronbach's alphas with

14 each item deleted all were less than 0.81 . The alphas of subscales ranged from acceptable to

15 good: "Beliefs that thoughts can cause illness, BI" $\alpha=0.76$; "Beliefs about biased thinking,

16 BT" $\alpha=0.72$; "Beliefs that thoughts are uncontrollable, BU" $\alpha=0.68$. The test-retest reliability

17 was tested by ICC. Based on the recommendation of Landis and Koch [42], an ICC of 0.1 or

18 lower was rated as no consistency, an ICC between 0.11 and 0.40 was rated as poor, an ICC

19 between 0.41 and 0.6 was rated as ordinary, an ICC between 0.61 and 0.80 was rated as mod-

20 erate, and an ICC above 0.8 was rated as good. The results showed that ICC was 0.70 for

21 CMCQ-HA total $(\mathrm{p}<0.001), 0.63$ for $\mathrm{BI}(\mathrm{p}<0.001), 0.52$ for $\mathrm{BT}(\mathrm{p}<0.001), 0.59$ for $\mathrm{BU}$

22 ( $\mathrm{p}<0.001)$, indicating an acceptable test-retest reliability for the CMCQ-HA [42].

23 Table 2 Inter-correlations between scales

\begin{tabular}{lllllll} 
& 1 & 2 & 3 & 4 & 5 & 6 \\
\hline 1.CMCQHAT & & & & & \\
$2 . \mathrm{BI}$ & $.797 * *$ & & & & \\
$3 . \mathrm{BT}$ & $.732 * *$ & $.377^{* *}$ & & & \\
\hline
\end{tabular}




\begin{tabular}{lllllll}
\hline $4 . \mathrm{BU}$ & $.752^{* *}$ & $.354^{* *}$ & $.433^{* *}$ & & \\
5.SHAI & $.466^{* *}$ & $.282^{* *}$ & $.321^{* *}$ & $.488^{* *}$ & & \\
6.MCQ30 & $.384^{* *}$ & $.292^{* *}$ & $.271^{* *}$ & $.371^{* *}$ & $.344^{* *}$ & \\
$7 . \mathrm{EPQ}$ & $.200^{* *}$ & $.111^{* *}$ & $.136^{* *}$ & $.224 * *$ & $.338^{* *}$ & $.307 * *$ \\
\hline
\end{tabular}

1 Notes: Data presented as Pearson's correlation coefficient (r). ** means $\mathrm{p}<0.01$ (two tailed).

2 CMCQHAT = Metacognitions about Health total; BI = Beliefs that Thoughts can Cause Ill-

3 ness; BT= Beliefs about biased Thinking; BU = Beliefs that Thoughts are Uncontrollable;

$4 \quad \mathrm{SHAI}=$ Short Health Anxiety Inventory; MCQ30 = Metacognition questionnaire-30; $\mathrm{EPQ}=$

5 Neuroticism subscale of the Eysenck Personality Questionnaire-Revised: Short Form.

\section{$6 \quad$ Factor structure}

7 The EFA was performed on scores from a randomly selected subsample $(n=596)$. The signifi-

8 cance of Bartlett's test of sphericity was observed $\left(\chi^{2}=2008.30, p<0.001\right)$. The Kai9 ser-Meyer-Olkin score was 0.84, which is considered good [43]. Both of those indices indicated that the factor analysis was appropriate. The inspection of the scree plot and eigenvalues

11 showed three factors in this analysis, which accounted for $51.05 \%$ of variance. The results of 12 the parallel analysis also showed three factors having eigenvalues above values obtained from 13 a random dataset. The first factor was well above the chance level (actual eigenvalue=2.493; 14 estimated mean $=0.216$; 95 th percentile eigenvalue $=0.250$ ), so as the second factor (actual ei15 genvalue $=2.345$; estimated mean $=0.172$; 95 th percentile eigenvalue $=0.201)$ and the third fac16 tor (actual eigenvalue $=1.881$; estimated mean $=0.134$; 95 th percentile eigenvalue $=0.167$ ). As shown in Table 3, there were five items which loaded highly on Factor 1 (beliefs about biased 18 thinking), factor 2 (beliefs that thoughts can cause illness), respectively. Meanwhile four 19 items loaded highly on Factor 3 (beliefs that thoughts are uncontrollable). The loading of each 20 item on its factor ranged from acceptable to good [35]. We conducted CFA on the remaining 21 members of the sample ( $\mathrm{n}=595$ ) using maximum likelihood estimation (MLM) to evaluate the 22 fitness of the EFA identified three-factor model. The MLM estimator reports a mean adjusted 23 chi-square (Satorra-Bentler $\chi^{2}$ ), which is appropriate for our data characterization. In general, 24 the cutoffs for acceptable fit are RMSEA with SRMR values of $\leqslant 0.08$, and TLI with CFI 
1 values of $\geqslant 0.90$ [37]. Results indicated that the three-factor model fit the data well: $\mathrm{SB} \chi^{2}=$

2 223.911, $\mathrm{df}=74, p<0.001, \mathrm{CFI}=0.930, \mathrm{TLI}=0.913, \mathrm{RMSEA}=0.052, \mathrm{SRMR}=0.049$.

3 Table 3 Exploratory factor analysis pattern matrix and structure matrix rotated to the Promax

4 criterion using principal axis factoring $(\mathrm{N}=596)$

\begin{tabular}{|c|c|c|c|c|c|c|}
\hline \multirow[t]{2}{*}{ Items } & \multicolumn{3}{|c|}{ Pattern Matrix } & \multicolumn{3}{|c|}{ Structure Matrix } \\
\hline & Factor 1 & Factor2 & Factor3 & Factor 1 & Factor2 & Factor3 \\
\hline 5 & 0.893 & -0.129 & -0.046 & 0.823 & 0.196 & 0.248 \\
\hline 6 & 0.682 & 0.047 & 0.055 & 0.724 & 0.350 & 0.367 \\
\hline 4 & 0.601 & 0.010 & -0.020 & 0.597 & 0.236 & 0.237 \\
\hline 9 & 0.521 & 0.164 & -0.043 & 0.568 & 0.344 & 0.272 \\
\hline 1 & 0.448 & 0.034 & 0.134 & 0.517 & 0.291 & 0.340 \\
\hline 14 & 0.011 & 0.741 & -0.016 & 0.297 & 0.735 & 0.431 \\
\hline 8 & -0.008 & 0.602 & 0.022 & 0.239 & 0.613 & 0.379 \\
\hline 3 & 0.019 & 0.577 & -0.017 & 0.240 & 0.574 & 0.335 \\
\hline 11 & -0.028 & 0.480 & 0.018 & 0.169 & 0.480 & 0.293 \\
\hline 10 & 0.075 & 0.440 & 0.033 & 0.262 & 0.489 & 0.327 \\
\hline 12 & -0.093 & 0.050 & 0.720 & 0.226 & 0.444 & 0.711 \\
\hline 7 & 0.074 & -0.037 & 0.653 & 0.331 & 0.382 & 0.661 \\
\hline 2 & 0.066 & -0.041 & 0.505 & 0.260 & 0.287 & 0.508 \\
\hline 13 & -0.011 & 0.076 & 0.461 & 0.211 & 0.347 & 0.502 \\
\hline
\end{tabular}

5 Convergent and divergent validity

6 As presented in Table 2, there was a significant positive correlation between total score of

7 CMCQ-HA and SHAI which measured the HA symptoms $(r=0.466, p<0.01)$. According to the

8 categorization used by Dancey and Reidy [44], an $\mathrm{r}$ below 0.40 was rated as a low correlation,

9 an $r$ between 0.40 and 0.60 was rated as moderate, and an $r$ above 0.60 was rated as strong.

10 The scores of three subscales also were significantly correlated with SHAI total score. Only

11 "Beliefs that thoughts are uncontrollable" showed correlations with SHAI above 0.40

12 ( $\mathrm{r}=0.488)$. These results supported the convergent validity of CMCQ-HA. Furthermore, the 
1 CMCQ-HA total was positively correlated with the SHAI $(r=0.466, p<0.01)$, which indicated

2 that the criterion-related validity was adequate. To examine the divergent validity, we calcu-

3 lated the Pearson's correlation values between CMCQ-HA total, SHAI total and EPQ-R-N.

4 Because neuroticism has been designated as a vulnerability of general anxiety both theoreti-

5 cally and clinically [45], we predicted that the correlation between CMCQ-HA and SHAI

6 would be stronger than correlations between CMCQ-HA and EPQ-R-N, the same as the results found in Bailey and Wells' study [20]. Our results revealed significant correlations existed between all the scales. Only the correlation between CMCQ-HA total and SHAI total was above 0.40, which was considered to be a moderate correlation [44] (see Table 2). Based on the results above, we used Steiger's $Z$ test to further examine whether the magnitude of the correlation between CMCQ-HA total and SHAI total ( $\mathrm{r}=0.466)$ was statistically significantly higher than the correlation between CMCQ-HA total and EPQ-R-N ( $r=0.338)$ [46]. The results suggested that the correlation between HA-related metacognitions and HA was significantly stronger than that between HA-related metacognitions and neuroticism $(\mathrm{Z}=8.665, p<0.001)$, as we predicted. Those results supported the divergent validity of CMCQ-HA.

\section{Incremental validity}

Because the CMCQ-HA is specially designed to measure of HA-related metacognitions, we explored whether CMCQ-HA could explain additional variance in HA over MCQ-30 with hierarchical multiple regression. The SHAI total was used as the dependent variable and all subscales of MCQ-30 were included in the model as a block (Model 1). Then the subscales of

21 CMCQ-HA were added to the model in the second step (Model 2). As presented in Table 4, there was $20.1 \%$ variance in SHAI explained by the subscales of MCQ-30 in the first model. The subscales of CMCQ-HA accounted for an additional $11.7 \%$ of the variance in SHAI in the second model. The variance explained by Model 2 was statistically significant and higher than Model $1(\Delta \mathrm{F}=67.600, p<0.05)$. In the final model, three CMCQ-HA subscales and two MCQ-30 subscales significantly contributed to the variance of SHAI: CMCQ-HA "Beliefs that thoughts can cause illness" $(\beta=0.061, p<0.05)$, CMCQ-HA "Beliefs about biased thinking" $(\beta=0.074, p<0.05)$, CMCQ-HA "Thoughts about illness are uncontrollable" $(\beta=0.322$, 
$1 p<0.05)$, MCQ-30 "Cognitive confidence" $(\beta=0.095, p<0.05)$, MCQ-30 "Uncontrollability

2 and Danger" $(\beta=0.208, p<0.05)$. Hence, the MCQ-HA is meaningfully different from the 3 MCQ-30.

4 Table 4 Hierarchical regression analyses of health related metacognitions as predictors of 5 health anxiety

\begin{tabular}{|c|c|c|c|c|c|}
\hline $\begin{array}{l}\text { Dependent } \\
\text { variable }\end{array}$ & Predictor & $\beta$ & $\mathrm{t}$ & $\Delta \mathrm{R}^{2}$ & $\Delta \mathrm{F}$ \\
\hline \multirow{16}{*}{$\begin{array}{l}\text { Health } \\
\text { anxiety }\end{array}$} & Step 1 & & & $0.201^{* *}$ & 59.683 \\
\hline & Cognitive confidence & $0.113^{* *}$ & 3.853 & & \\
\hline & Positive Beliefs & 0.050 & 1.606 & & \\
\hline & Cognitive self-consciousness & -0.032 & -1.040 & & \\
\hline & Uncontrollability and Danger & $0.368^{* *}$ & 11.990 & & \\
\hline & Need to control thoughts & 0.031 & 0.992 & & \\
\hline & Step 2 & & & $0.117^{* *}$ & 67.600 \\
\hline & Cognitive confidence & $0.095^{* *}$ & 3.488 & & \\
\hline & Positive Beliefs & 0.037 & 1.277 & & \\
\hline & Cognitive self-consciousness & -0.027 & -0.956 & & \\
\hline & Uncontrollability and Danger & $0.208^{* *}$ & 6.818 & & \\
\hline & Need to control thoughts & 0.004 & 0.127 & & \\
\hline & Beliefs that Thoughts can Cause Ill- & $0.061^{*}$ & 2.285 & & \\
\hline & ness & & & & \\
\hline & Beliefs about biased Thinking & $0.074^{* *}$ & 2.671 & & \\
\hline & $\begin{array}{l}\text { Beliefs that Thoughts are Uncontrolla- } \\
\text { ble }\end{array}$ & $0.322^{* *}$ & 11.134 & & \\
\hline
\end{tabular}

\section{$7 \quad$ Invariance and difference across gender}

8 The maximum likelihood estimation (MLM) estimator was also performed in this analysis. To 
1 test the measurement invariance across gender, we first conducted CFA in each gender group

2 separately to confirm the three-factor structure of CMCQ-HA. As shown in Table 5: all TLI

3 and CFI were above 0.90; the RMSEA values and SRMR values were below 0.08 . Thus the

4 structure proposed by Bailey and Wells [20] fit the data well for each gender group. We tested

5 the four degrees of measurement invariance across gender step by step. As presented in Table

65 , the data fit every model well. In different consecutive models, the following indices were

7 used to evaluate the invariance: the changes in CFI ( $\Delta \mathrm{CFI})$, the Bayesian information criterion

8 (BIC) value. A $\triangle \mathrm{CFI} \leq 0.010$ and a descending BIC value was regarded as evidence of invar-

9 iance across gender [47]. The results showed that the $\Delta$ CFI were below 0.01 and the BIC de-

10 creased between any two models. In comparing the metric model to the configural model, the

$11 \Delta \mathrm{CFI}=0.001$, the BIC decreased by 63.492 ; comparing scalar model to metric model, the

$12 \Delta \mathrm{CFI}=-0.004$, the BIC decreased by 44.793 ; in comparing the strict model to the scalar

13 model, the $\triangle \mathrm{CFI}=-0.04$, the BIC decreased by 49.183 . Taking those findings together, the

14 measurement invariance was tenable across both gender groups. The confirmation of strict

15 invariance means that the difference among observed scores' variance was exactly the differ-

16 ence among latent variables' variance [48]. Based on that, we conducted a t-test to explore

17 whether a difference existed in CMCQ-HA scores between men and women. The results in-

18 dicated that $\mathrm{s}$ no significant difference $(\mathrm{p}>0.05)$ existed between the two gender groups in

19 CMCQ-HA total score and the three subscale scores (see Table 6).

20 Table 5 Fit indices of measurement invariance for the CMCQ-HA across gender

\begin{tabular}{|c|c|c|c|c|c|c|c|c|c|}
\hline Model & $\mathrm{S}-\mathrm{B} \chi^{2}$ & $\mathrm{df}$ & TLI & CFI & $\operatorname{RMSEA}(90 \% \mathrm{CI})$ & SRMR & $\begin{array}{l}\text { Model } \\
\text { comparison }\end{array}$ & $\Delta \mathrm{CFI}$ & $\mathrm{BIC}$ \\
\hline Male $(n=465)$ & 171.526 & 74 & 0.933 & 0.946 & $0.046(0.035-0.057)$ & 0.051 & & & \\
\hline Female $(n=726)$ & 228.270 & 74 & 0.922 & 0.937 & $0.048(0.040-0.056)$ & 0.041 & & & \\
\hline Model1 & 399.778 & 148 & 0.927 & 0.940 & $0.047(0.041-0.054)$ & 0.045 & & & 36679.127 \\
\hline Model2 & 414.220 & 159 & 0.932 & 0.941 & $0.045(0.039-0.052)$ & 0.048 & 2 vs. 1 & 0.001 & 36615.635 \\
\hline Model3 & 433.161 & 170 & 0.932 & 0.937 & $0.046(0.039-0.052)$ & 0.048 & 3 vs. 2 & -0.004 & 36570.842 \\
\hline Model4 & 483.120 & 184 & 0.933 & 0.933 & $0.045(0.039-0.051)$ & 0.051 & 4 vs. 3 & -0.004 & 36521.659 \\
\hline
\end{tabular}


1 Notes: Model1=configural invariance; Model2=metric invariance; Model3=scalar invariance;

2 Model4= strict invariance; $\mathrm{S}-\mathrm{B} \chi^{2}=$ Satorra-Bentler scaled $\chi^{2} ; \mathrm{df}=$ degrees of freedom; TLI =

3 the Tuker-Lewis index; CFI = Comparative Fit index; RMSEA= root-mean-square error of

4 approximation; SRMR = standardized root mean squared residual; BIC $=$ Bayesian infor-

5 mation criterion.

6 Table 6 T-test for scores of CMACQ-HA between two gender group

\begin{tabular}{|c|c|c|c|c|}
\hline & \multicolumn{2}{|c|}{ Mean(SD) } & \multirow[t]{2}{*}{$\mathrm{t}$} & \multirow[t]{2}{*}{$\mathrm{p}$} \\
\hline & Female $(n=726)$ & $\operatorname{Male}(n=465)$ & & \\
\hline CMCQHAT & $24.437(6.048)$ & $24.341(6.374)$ & -0.263 & 0.792 \\
\hline BI & $10.207(3.302)$ & $10.293(3.175)$ & 0.446 & 0.656 \\
\hline BT & $7.316(2.180)$ & $7.237(2.606)$ & -0.546 & 0.585 \\
\hline $\mathrm{BU}$ & $6.915(2.470)$ & $6.811(2.475)$ & -0.703 & 0.482 \\
\hline
\end{tabular}

7 Notes: $\mathrm{CMCQHAT}=$ Metacognitions about Health total; BI $=$ Beliefs that Thoughts can

8 Cause Illness; BT= Beliefs about biased Thinking; BU $=$ Beliefs that Thoughts are Uncon-

9 trollable.

10 Discussion

11 This study describes the development of the CMCQ-HA in a sample of more than 1000 col-

12 lege students, undertaken in order to better measure the psychological mechanism of health

13 anxiety (HA) in a Chinese sample in mainland China. The quality of data in this study was

14 satisfactory; the effective return ratio was $92.3 \%$ and no missing data existed for the remain-

15 ing 1191 respondents. Low to moderate floor effects were found in our study. One possible

16 explanation for it is the characteristics of our sample. As our participants were selected from

17 college students which have a lower level of health anxiety compared with the clinic sample,

18 most respondents scored at the positive pole of MCQ-HA.

19 The Psychometric characteristics, including reliability and validity, were tested in the total

20 sample and across gender. The CMCQ-HA showed an acceptable to good reliability with a

21 Cronbach's alpha value 0.81 for total and $0.68-0.76$ for three subscales. Equally, good stability

22 was also confirmed by its test-retest reliability which estimated by ICC (ICCs higher than 
1 0.41). The results of Exploratory Factor Analysis (EFA) indicated that CMCQ-HA has the

2 same three factors as reported by Bailey and Wells [20]. Then the structure of the three factors

3 was further confirmed by Confirmatory Factor Analysis (CFA). Moreover, good convergent

4 and discriminant validity was confirmed by correlations between CMCQ-HA total score,

5 CMCQ-HA subscale scores, SHAI total score and the EPQ-R-N. As expected, the total score

6 of CMCQ-HA were positively correlated with the SHAI total score, and these correlations

7 were significantly stronger than the correlations between CMCQ-HA total score and the

8 EPQ-R-N, indicating that the metacognitive beliefs measured by the CMCQ-HA are specially

9 related to HA but not to general anxiety. That result is consistent with findings from previous

10 studies [20], with the same situation was observed for incremental validity. To estimate the

11 incremental validity, we explored the possibility of the CMCQ-HA accounted for additional

12 variance in HA over and above the variance in HA explained by general metacognitions as

13 measured by the MCQ-30. In the final regression model, CMCQ-HA subscales explained an

14 extra variance of $11.7 \%$ over and above MCQ-30 subscales.

15 Overall our findings supported the metacognitive model which indicated that some specific 16 metacognitive beliefs have positive correlations with HA symptoms. However the correlation coefficients between HA and the CMCQ-HA total and subscale scores were a little lower than coefficients reported by Bailey and Wells. In Bailey's research [20], the correlation coefficient between MCQ-HA total and HA was 0.693 compared to 0.466 in the present study, and the correlation coefficients between MCQ-HA subscale scores and HA ranged from 0.486-0.711 compared to $0.282-0.488$ in our study. Only one subscale "Beliefs that thoughts are uncontrollable" showed a moderate correlation with SHAI, with the others showing significant but weak correlations, whereas in the previous study all three subscales showed moderate correlations with HA [20]. A reason for this result may be attributed to the different measures used to assess HA in the two studies. Bailey and Wells [20] used the Whiteley Index to measure HA while the SHAI was used in the current study. These two measures may vary with regard to their degree of efficacy in assessing the level of HA. Items in the Whiteley Index are based on the symptom clusters of hypochondriasis [12], while the SHAI was designed according to the 
1 cognitive theory of HA [13]. Such results suggest that the key content of HA or hypochondri-

2 asis and the mechanism of symptom maintenance need further exploration. The possible cultural diversity in metacognition may be another reason for this. The baseline level of

4 HA-related metacognition in people from different cultures may be not the same; or people

5 may hold particular HA-related metacognition in some cultures. Future researches should do

6 more comparisons across culture. We also tested the measurement invariance of the

7 CMCQ-HA across gender. The configural, metric, scalar and strict invariance all were estab-

8 lished. Then we compared the scores of CMCQ-HA between the two gender groups, and no

9 statistically significant difference was found. That result further supported the validity of the 10 CMCQ-HA.

11 There are some limitations in current study. First, the Chinese study participants are a con12 venience sample recruited from a single university and were all medical students. As such, 13 they were predominantly young, female, and without physical diseases. Further studies based 14 on more randomly selected Chinese population samples as well as a clinical sample are 15 needed to establish the generalisability of this measure. Second, we used only two scales for 16 the validity estimation. Because the key content of HA is controversial, a variety of measures 17 about HA should be included to consider alternative theories of HA. Finally, we used only the 18 SHAI for evaluating a limited number of criterion variables. Evaluations of more relevant 19 criterion variables with other measures are needed for further research.

20 In conclusion, sufficient reliability and validity of CMCQ-HA were confirmed in a Chinese 21 college student sample. More important, this study presented further evidence for the metacognitive model of HA in a different culture. The CMCQ-HA will be a promising tool to assess HA-related metacognitions in China, and to enhance understanding of the link between metacognitive beliefs and HA. tal of Central South University (grant no.20150302). 
3 Declaration of Conflicting Interests The authors declared no potential conflicts of interests with respect to the research, authorship, and/or publication of this article.

6 Ethical approval The Institutional Review Board of the Third Xiangya Hospital in Hunan approved the study (2017-S208).

\section{Appendix}

10 The Metacognitions about Health Questionnaire

\begin{tabular}{|l|l|l|l|l|}
\hline Items & $\begin{array}{l}\text { Do not } \\
\text { agree }\end{array}$ & $\begin{array}{l}\text { Agree } \\
\text { slightly }\end{array}$ & $\begin{array}{l}\text { Agree } \\
\text { moderately }\end{array}$ & $\begin{array}{l}\text { Agree } \\
\text { very } \\
\text { much }\end{array}$ \\
\hline 1. Thinking of illness could change my health. & 1 & 2 & 3 & 4 \\
\hline $\begin{array}{l}\text { 2. I cannot have peace of mind so long as I have } \\
\text { physical symptoms. }\end{array}$ & 1 & 2 & 3 & 4 \\
\hline $\begin{array}{l}\text { 3. I will be punished for thinking I am in good } \\
\text { health. }\end{array}$ & 1 & 2 & 3 & 4 \\
\hline $\begin{array}{l}\text { 4. Thinking negatively can increase my chances } \\
\text { of disease. }\end{array}$ & 1 & 2 & 3 & 4 \\
\hline $\begin{array}{l}\text { 5. Worrying about illness is likely to make it } \\
\text { happen. }\end{array}$ & 1 & 2 & 3 & 4 \\
\hline $\begin{array}{l}\text { 6. Some thoughts have the power to make me ill. } \\
\text { 7. Dwelling on thoughts of illness is uncontrol- }\end{array}$ & 1 & 2 & 3 & 4 \\
\hline \begin{tabular}{l} 
lable. \\
\hline $\begin{array}{l}\text { 8. Thinking the worse about symptoms will keep } \\
\text { me safe. }\end{array}$
\end{tabular} & 1 & 2 & 3 & 4 \\
\hline $\begin{array}{l}\text { 9. Worrying about my health will damage my } \\
\text { body. }\end{array}$ & 1 & 2 & 3 & 4 \\
\hline $\begin{array}{l}\text { 10. If I think positively about physical symptoms } \\
\text { I will be caught off guard. }\end{array}$ & 1 & 2 & 3 & 4 \\
\hline $\begin{array}{l}\text { 11. Worrying about my health will help me cope. } \\
\text { 12. I have no control over thinking about my } \\
\text { health. }\end{array}$ & 1 & 2 & 3 & 3 \\
\hline 13. Only if I have a diagnosis will I be able to & 1 & 2 & 3 & 4 \\
\hline
\end{tabular}




\begin{tabular}{|l|l|l|l|l|}
\hline stop worrying. & & & & \\
\hline $\begin{array}{l}\text { 14. Thinking positively about my health will } \\
\text { tempt fate and I will become ill. }\end{array}$ & 1 & 2 & 3 & 4 \\
\hline
\end{tabular}

2 健康焦虑元认知量表中文版

\begin{tabular}{|c|c|c|c|c|}
\hline 条目 & 不同意 & $\begin{array}{l}\text { 有点同 } \\
\text { 意 }\end{array}$ & $\begin{array}{l}\text { 基 本 同 } \\
\text { 意 }\end{array}$ & $\begin{array}{l}\text { 完全 同 } \\
\text { 意 }\end{array}$ \\
\hline 1. 对疾病的思考会影响我的健康状况。 & 1 & 2 & 3 & 4 \\
\hline $\begin{array}{l}\text { 2.只要我有身体症状, 我就不能保持平和 } \\
\text { 心态。 }\end{array}$ & 1 & 2 & 3 & 4 \\
\hline $\begin{array}{l}\text { 3.如果我认为自己健康状况很好, 那么可 } \\
\text { 能会发生一些不好的事。 }\end{array}$ & 1 & 2 & 3 & 4 \\
\hline 4.消极地思考会增加我患病的可能性。 & 1 & 2 & 3 & 4 \\
\hline 5.对疾病的担心有可能引发疾病。 & 1 & 2 & 3 & 4 \\
\hline 6.有些与疾病有关的想法会导致我生病。 & 1 & 2 & 3 & 4 \\
\hline $\begin{array}{l}\text { 7.思考与疾病有关的想法是我无法控制 } \\
\text { 的。 }\end{array}$ & 1 & 2 & 3 & 4 \\
\hline 8.我把症状想得更严重就会更安全 & 1 & 2 & 3 & 4 \\
\hline $\begin{array}{l}\text { 9.对自己健康的担忧会损害我的身体健 } \\
\text { 康 }\end{array}$ & 1 & 2 & 3 & 4 \\
\hline $\begin{array}{l}\text { 10.如果我太乐观地看待我的身体症状, } \\
\text { 我将会意识不到疾病的存在 }\end{array}$ & 1 & 2 & 3 & 4 \\
\hline 11.对疾病的担忧会帮助我应对疾病。 & 1 & 2 & 3 & 4 \\
\hline 12.我无法控制自己对自身健康的思考。 & 1 & 2 & 3 & 4 \\
\hline $\begin{array}{l}\text { 13. 只有当我得到了一个诊断, 我才能停 } \\
\text { 止对自己身体状况的担忧。 }\end{array}$ & 1 & 2 & 3 & 4 \\
\hline $\begin{array}{l}\text { 14.如果我对自身健康持乐观的想法, 我 } \\
\text { 是会因此生病的 }\end{array}$ & 1 & 2 & 3 & 4 \\
\hline
\end{tabular}

\section{References:}

1. Abramowitz, J. S., Deacon, B. J. \& Valentiner, D. P. (2007). The short health anxiety inventory: Psychometric properties and construct validity in a non-clinical sample. Cognitive Therapy and Research, 31(6), 871-883. doi: 10.1007/s10608-006-9058-1

2. Creed, F. \& Barsky, A. (2004). A systematic review of the epidemiology of somatisation disorder and hypochondriasis. Journal of Psychosomatic Research, 56(4), 391-408. doi: 
3. Salkovskis, P. M. \& Warwick, H. M. (1986). Morbid preoccupations, health anxiety and reassurance: a cognitive-behavioural approach to hypochondriasis. Behaviour Research and Therapy, 24(5), 597-602

4. Sunderland, M., Newby, J. M. \& Andrews, G. (2013). Health anxiety in Australia: prevalence, comorbidity, disability and service use. The British Journal of Psychiatry, 202(1), 56-61. doi: 10.1192/bjp.bp.111.103960

5. Noyes, R., Happel, R. L. \& Yagla, S. J. (1999). Correlates of hypochondriasis in a nonclinical population. Psychosomatics, 40, 461-469

6. Malouff, J. M., Thorsteinsson, E. B. \& Schutte, N. S. (2005). The relationship between the five-factor model of personality and symptoms of clinical disorders: A meta-analysis. Journal of Psychopathology and Behavioral Assessment, 27, 101-114

7. Cox, B. J., Borger, S. C., Asmundson, G. J. G. \& Taylor, S. (2000). Dimensions of hypochondriasis and the five factor model of personality. Personality and individual differences, 29, 99-108

8. Williams, P. G. (2004). The psychopathology of self-assessed health: A cognitive approach to health anxiety and hypochondriasis. Cognitive Therapy Research, 28(5), 629-644

9. Marcus, D. K., Gurley, J. R., Marchi, M. M. \& Bauer, C. (2007). Cognitive and perceptual variables in hypochondriasis and health anxiety: A systematic review. Clinical psychology review, 27(2), 127-139. doi: 10.1016/j.cpr.2006.09.003

10. L Norris, A. \& Marcus, D. K. (2014). Cognition in health anxiety and hypochondriasis: recent advances. Current Psychiatry Reviews, 10(1), 44-49

11. Barsky, A. J. \& Wyshak, G. (1990). Hypochondriasis and somatosensory amplification. The British Journal of Psychiatry, 157(3), 404-409

12. Pilowsky, I. (1967). Dimension of hypochondriasis. British Journal of Psychiatry, 113, 89-93

13. Salkovskis, P. M., Rimes, K. A., Warwick, H. \& Clark, D. M. (2002). The Health Anxiety Inventory: development and validation of scales for the measurement of health anxiety and hypochondriasis. Psychological Medicine, 32(5), 843-853. doi: 10.1017/S0033291702005822

14. Hadjistavropoulos, H. D., Janzen, J. A., Kehler, M. D., Leclerc, J. A., Sharpe, D. \& Bourgault-Fagnou, M. D. (2012). Core cognitions related to health anxiety in self-reported medical and non-medical samples. Journal of Behavioral Medicine, 35(2), 167-178. doi: 10.1007/s10865-011-9339-3

15. Bailey, R. \& Wells, A. (2016). Is metacognition a causal moderator of the relationship between catastrophic misinterpretation and health anxiety? A prospective study. Behaviour research and therapy, 78, 43-50. doi: 10.1016/j.brat.2016.01.002

16. Bailey, R. \& Wells, A. (2016). The contribution of metacognitive beliefs and dysfunctional illness beliefs in predicting health anxiety: An evaluation of the metacognitive versus the cognitive models. Clinical Psychologist, 20(3), 129-137. doi: 10.1111/cp.12078

17. Bailey, R. \& Wells, A. (2015). Metacognitive beliefs moderate the relationship between catastrophic misinterpretation and health anxiety. Journal of Anxiety Disorders, 34, 8-14. doi: 10.1016/j.janxdis.2015.05.005

18. Melli, G., Carraresi, C., Poli, A. \& Bailey, R. (2016). The role of metacognitive beliefs in health anxiety. Personality and Individual Differences, 89, 80-85. doi: 10.1016/j.paid.2015.10.006

19. Wells, A. \& Cartwright-Hatton, S. (2004). A short from of the metacognitions questionnaire: prop- 
erties of the MCQ-30. Behaviour research and therapy, 42(4), 385-396

20. Bailey, R. \& Wells, A. (2015). Development and initial validation of a measure of metacognitive beliefs in health anxiety: The MCQ-HA. Psychiatry research, 230(3), 871-877. doi: 10.1016/j.psychres.2015.11.035

21. Rachman, S. (2012). Health anxiety disorders: a cognitive construal. Behav Res Ther, 50(7-8), 502-512. doi: 10.1016/j.brat.2012.05.001

22. Fulton, J. J., Marcus, D. K. \& Merkey, T. (2011). Irrational health beliefs and health anxiety. J Clin Psychol, 67(6), 527-538. doi: 10.1002/jclp.20769

23. Looper, K. J. \& Kirmayer., L. J. (2001). Hypochondriacal concerns in a community population. Psychological Medicine, 31(4), 577-584

24. Lee, S., Ng, K. L., Ma, Y. L., Tsang, A. \& Kwok, K. P. (2011). A general population study of the Chinese Whiteley-7 index in Hong Kong. J Psychosom Res, 71(6), 387-391. doi: 10.1016/j.jpsychores.2011.05.013

25. Su, X., Li, Z., Jiang, C. \& Ma, Y. (2016). The cognitive characteristics of hypochondriasis and the development of related measurements. Journal of Psychiatry, 29(6), 477-480

26. Markus, H. R. \& Kitayama, S. (1991). Culture and the self: Implications for cognition, emotion, and motivation. Psychological review, 98(2), 224-253

27. Beaton, D. E., Bombardier, C., Guillemin, F. \& Ferraz, M. B. (2000). Guidelines for the process of cross-cultural adaptation of self-report measures. Spine (Phila Pa 1976), 25(24), 3186-3191

28. Yuan, Y., Zhang, Y., Liu, R., Li, G. \& Mao, S. (The reliability and validity of a Chinese-version Short Health Anxiety Inventory: an investigation of university students. Neuropsychiatric Disease and Treatment, 1739. doi: 10.2147/NDT.S83501

29. Cartwright-Hatton, S. \& Wells, A. (1997). Beliefs about worry and intrusions: the metacognitions questionnaire and its correlates. Journal of Anxiety Disorders, 24(3), 318-325

30. Carciofo, R., Song, N., Du, F., Wang, M. M. \& Zhang, K. (2017). Metacognitive beliefs mediate the relationship between mind wandering and negative affect. Personality and Individual Differences, 107, 78-87. doi: 10.1016/j.paid.2016.11.033

31. Eysenck, S. B. G., Eysenck, H. J. \& Barrett, P. (1985). A revised version of the psychoticism scale. Personality and individual differences, 6(1), 21-29

32. Mingyi, Q., Guocheng, W., Rongchun, Z. \& Shen, Z. (2000). Development of the revised Eysenck Personality Questionnaire Short Scale for Chinese (EPQ-RSC). Acta Psychologica Sinica, 32(3), 317-323

33. Naunnally, J. C. \& Bernstein, I. H. (1994). Psychometric Theory, third ed. New York: McGraw-Hill.

34. Shrout, P. E. \& Fleiss, J. L. (1979). Intraclass correlations: uses in assessing rater reliability. Psychological bulletin, 86(2), 420-428

35. Tabachnick, B. G. \& Fidell, L. S. (2007). Using Multivariate Statistics, fifth ed. New York: Allyn and Bacon.

36. O'Connor, B. P. (2000). SPSS and SAS programs for determining the number of components using parallel analysis and Velicer's MAP test. Behavior Research Methods, 32(3), 396-402

41 37. Hu, L. T. \& Bentler, P. M. (1999). Cutoff criteria for fit indexes in covariance structure analysis: Conventional criteria versus new alternatives. Structural Equation Modeling: A Multidisciplinary 
Journal, 6(1), 1-55

38. Samuel, D. B., South, S. C. \& Griffin, S. A. (2015). Factorial invariance of the five-factor model rating form across gender. Assessment, 22(1), 65-75. doi: 10.1177/1073191114536772

39. Kline, R. B. (2005). Principles and practice of structural equation modeling. New York, Guilford.

40. Ambrosio, L., Portillo, M. C., Rodriguez-Blazquez, C., Martinez-Castrillo, J. C., Rodriguez-Violante, M. \& Serrano-Duenas, M., et al. (2016). Satisfaction with Life Scale (SLS-6): First validation study in Parkinson's disease population. Parkinsonism Relat Disord, 25, 52-57. doi: 10.1016/j.parkreldis.2016.02.012

41. Zwaanswijk, W., Veen, V. C. \& Vedder, P. (2016). The Youth Psychopathic Traits Inventory: A Bifactor Model, Dimensionality, and Measurement Invariance. Assessment. doi: $10.1177 / 1073191116632340$

42. Shrout, P. E. (1998). Measurement reliability and agreement in psychiatry. Statistical methods in medical research, 7(3), 301-317

14 43. Kaiser, H. F. (1970). A Second Generation Little Jiffy. Psychometrika, 35(4), 401-415

15 44. Dancey, C. \& Reidy, J. (2004). Statistics without maths for psychology: Using SPSS for windows. London: Prentice Hall.

17 45. Enns, M. W. \& Cox, B. J. (1997). Personality dimensions and depression: review and commentary. The Canadian Journal of Psychiatry, 42(3), 274-284. doi: 10.1177/070674379704200305

46. Steiger, J. H. (1980). Tests for comparing elements of a correlation matrix. Psychological Bulletin, $87,245-251$

47. Cheung, G. W. \& Rensvold, R. B. (2002). Evaluating goodness-of-fit indexes for testing measurement invariance. Structural equation modeling, 9(2), 233-255. doi: 10.1207/S15328007SEM0902_5

48. Schmitt, N. \& Kuljanin, G. (2008). Measurement invariance: Review of practice and implications. 